\title{
APRENDIZAJE DE VOCABULARIO EN L2: LA RELACIÓN ENTRE LA VARIABLE SEXO Y LA EFECTIVIDAD DE ACTIVIDADES DE RECONOCIMIENTO Y DE PRODUCCIÓN. RESULTADOS PRELIMINARES
}

\author{
Alicia San-Mateo-Valdehíta \\ Universidad Nacional de Educación a Distancia \\ asanmateo@flog.uned.es
}

\section{RESUMEN}

Este trabajo recoge los resultados de una investigación sobre la eficacia de actividades de reconocimiento y de producción encaminadas al aprendizaje de vocabulario en español como L2, en relación con la variable sexo. 150 estudiantes de español de nivel B1, según el MCER (Consejo de Europa, 2002), 117 mujeres y 33 hombres, divididos en tres grupos, practican con un ejercicio diferente; dos de reconocimiento: (1) selección de definiciones y (2) selección de ejemplos; y uno de producción: (3) escritura de oraciones. El análisis de los resultados indica, por un lado, que la diferencia entre los promedios alcanzados por hombres y mujeres no es significativa, independientemente del tipo de tarea de instrucción; y, por otro, que ellas consiguen recordar un número significativamente mayor de palabras con el ejercicio de escritura que con el de selección de definiciones; en cambio, en ellos los resultados obtenidos con las tres tareas no son significativamente diferentes. Estos resultados se analizan teniendo en cuenta las estrategias de aprendizaje puestas en práctica.

Palabras Clave: Aprendizaje de vocabulario, actividad de reconocimiento, actividad de producción, variable sexo, estrategia de aprendizaje.

\section{ABSTRACT}

This paper presents the results of research on the effectiveness of recognition and production activities on learning vocabulary in Spanish as L2, taking into account the gender 
variable. 150 B1 level (MCER or CEFR, in English, Consejo de Europa, 2002) students of Spanish, 33 males and 117 females, divided into three groups, practised with a different task; two recognition activities: (1) selection of definitions and (2) selection of examples; and one production activity: (3) writing sentences. The analysis of results indicates, first, that the gender variable is not significant, regardless of the instruction task. Second, females manage to recall significantly more words when they practise writing sentences than when choosing definitions; nevertheless, males do not achieve different results significantly with any of three tasks. Results are analyzed taking into account the learning strategies implemented.

KEYWORDS: Vocabulary learning, recognition activity, production activity, gender, learning strategies.

\section{INTRODUCCIÓN}

La diferencia entre los resultados que alcanzan los aprendices de L2 o el nivel de dominio que consiguen se ha tratado de explicar por diversos motivos, entre ellos la edad a la que se inicia el aprendizaje, la aptitud lingüística, factores socio-psicológicos como la motivación o la actitud hacia la lengua; la personalidad, el estilo cognitivo, la especialización de los hemisferios cerebrales o las estrategias de aprendizaje empleadas; y otros factores como la memoria, la voluntad, el interés, el dominio de la propia L1, las alteraciones lingüísticas, como la dislexia o la estrefosimbolia, o el sexo (Larsen-Freeman y Long, 1994:138-198). En este estudio vamos a centrarnos en ese último factor, el sexo, relacionándolo con el aprendizaje de vocabulario en la L2 (en este caso, español) con varios tipos de actividades, lo cual nos proporcionará información sobre las diferentes estrategias de aprendizaje empleadas por los sujetos.

El clásico estudio de Lakoff (1973) da cuenta de las diferencias entre el discurso de hombres y mujeres, reflejo de los papeles sociales que desempeñan cada uno y de las desigualdades sufridas por ellas — téngase presente el contexto en el que se escribió-. El uso de determinados nombres de colores (p. ej., mauve, ecru o beige), de partículas expletivas (p. ej., damn o funge), de adjetivos valorativos (p. ej., adorable, charming, sweet, lovely o divi$n e$ ), al igual que el empleo de preguntas de confirmación al final de una oración enunciativa (John is here, isn't he?) o de peticiones convertidas en interrogativas negativas (Won't you close the door?) parece ser casi exclusivo —o al menos más abundante- en el discurso de las mujeres. Añade la autora que en la enseñanza de L2 no se debe olvidar que el contexto social es relevante y el profesor debe ser consciente de la lengua que enseña, que es algo más que gramática, fonética y semántica:

[...] if a woman teacher unconsciously teaches 'women's language' to her male students, they may be in difficulties when they try to function in another country; if a female anthropologist learns the 'men's language' of an area, she may not be able to get anywhere with the inhabitants because she seems unfeminine, and they will not know how to react to her (Lakoff, 1973:76).

El profesor tiene que saber que la competencia semántica del aprendiz — que es, al fin y al cabo, de lo que nos ocupamos en este estudio - será necesariamente diferente en función de su edad, su desarrollo cognitivo, su experiencia del mundo y, también, del sexo (Jiménez Catalán, 2002:161). 
En el terreno de la L2 (véase Bowden, Sanz y Stafford, 2005:111-112), la mayor parte de los estudios revela que las mujeres consiguen mejores resultados en casi todos los ejercicios, aunque también hay investigaciones en las que los hombres alcanzan mayores logros, o en las que no hay diferencias significativas. Yazdanpanah (2007:71-73), en un trabajo sobre comprensión lectora, concluye que las puntuaciones que obtienen los hombres y las mujeres son diferentes dependiendo de la tarea que realicen. Ellas destacan cuando hay que identificar la idea principal del texto, adivinar el significado por el contexto y responder preguntas sobre coherencia (solo en estos dos últimos casos la diferencia con las puntuaciones de los hombres es significativa). En cambio, ellos sobresalen cuando hay que extraer datos específicos, identificar información y relacionar un párrafo con su título. En el estudio de Farhady (1982: 47), las mujeres solo consiguen puntuaciones significativamente superiores en el ejercicio de comprensión auditiva; en el resto (los de rellenar huecos, dictado, comprensión lectora y gramática), la diferencia no es significativa. Arifuddin, Susanto y Suharsono (2013:39-70) estudian los errores cometidos en un ejercicio de comprensión auditiva del examen TOEFL (Test Of English as a Foreign Language) y llegan a la conclusión de que, básicamente, no hay diferencias entre hombres y mujeres.

Por otro lado, en los trabajos sobre el manejo de estrategias de aprendizaje, también se llega a conclusiones diversas. P. ej., Bacon (1992:160) afirma que en su experimento sobre comprensión auditiva de material real con aprendices de español como L2, las mujeres emplean más estrategias metacognitivas, mientras que los hombres recurren más a las cognitivas ${ }^{1}$. Phakiti (2003:665-675), por su parte, en una investigación sobre comprensión lectora, afirma que no hay diferencia entre el dominio que demuestran hombres y mujeres ni en el uso que hacen de las estrategias cognitivas; en cambio, los hombres utilizan más estrategias metacognitivas que las mujeres — justo al contrario que en estudio de Bacon (1992)—. Salem (2006: 65-66) no observa diferencias significativas entre hombres y mujeres en relación con la motivación, con el dominio de la L2, ni en el empleo de estrategias de aprendizaje; en cambio, sí que parece que las mujeres usan significativamente más estrategias cognitivas, metacognitivas, de compensación y de memoria. En el estudio de Zeynali (2012:1616-1618), las mujeres se valen más tanto de las estrategias de aprendizaje como de otras estrategias sociales y afectivas, pero no hay diferencias con respecto a los hombres en cuanto al empleo de estrategias cognitivas y metacognitivas. En cambio, Radwan (2011:131-132) concluye que los hombres manejan más estrategias sociales que las mujeres; aunque, en la mayor parte de los estudios (véanse las revisiones de Oxford, Nyikos y Ehrman, 1988:326; y de Jiménez Catalán, 2003:56-57), la conclusión es que son precisamente las mujeres las que hacen mayor uso de las estrategias sociales. Por otro lado, Sankarakumar, Chandrakanthi y Malathy (2012:315-318), en la línea de Yazdanpanah (2007), confirman que, en las actividades de comprensión lectora, hombres

\footnotetext{
${ }^{1}$ Las estrategias metacognitivas son las que sirven para supervisar conscientemente el proceso de aprendizaje, e incluyen la planificación, el seguimiento y la evaluación de la actividad de aprendizaje; las cognitivas implican la manipulación de información en una tarea intermedia cuyo objetivo es el aprendizaje; p. ej., el análisis, la transformación o la síntesis del material, llevados a cabo por inferencia, deducción de significados por el contexto, elaboración de la información o relación de nuevos conceptos con otros ya conocidos. Las estrategias sociales tienen que ver con la interacción con otras personas para mejorar el conocimiento de la L2; son pedir aclaraciones o verificar lo aprendido; cooperar y empatizar con los demás hablantes, a través del conocimiento de su cultura; y las estrategias afectivas sirven para controlar las emociones, las actitudes y las motivaciones (O’Malley y Chamot, 1990: 44-47; Oxford, 1990:135-148).
} 
y mujeres utilizan estrategias diferentes, por lo que los resultados que obtienen difieren según el tipo de ejercicio: de opción múltiple, de verdadero o falso, o de emparejar una palabra del texto con un sinónimo; pero en este último, las diferencias no son significativas.

La disparidad que encontramos en los resultados sobre las posibles diferencias tanto en el domino que demuestran hombres y mujeres como en el uso de estrategias nos llevan a plantearnos la necesidad de poner en práctica más estudios en otros contextos y condiciones de aprendizaje.

\section{ESTRATEGIAS Y TÉCNICAS DE APRENDIZAJE Y LA VARIABLE SEXO}

En la mayor parte de las investigaciones sobre léxico en L2 el objetivo es explicar el proceso de desarrollo y ampliación del vocabulario en el aprendiz y para ello se trata de determinar la diferencia entre vocabulario activo (productivo) y pasivo (receptivo), analizar los errores léxicos detectados y las estrategias utilizadas — de las que hablaremos ahora- y estudiar el efecto de diversas técnicas de aprendizaje de vocabulario (Maiguashca, 1993), con el fin de saber con qué tipo de ejercicio el aprendiz incorpora un número mayor de palabras a su lexicón mental.

Schmitt (1997:206-217) elabora una clasificación de las estrategias de aprendizaje de vocabulario exclusivamente, pues las propuestas anteriores no las recogen de manera conveniente o es posible situarlas en más de un apartado. Las divide en dos grandes grupos: (1) de descubrimiento del significado, ya sea el aprendiz por sí mismo (análisis de las partes del discurso, de las partes de la palabra; uso del diccionario bilingüe o monolingüe, de tarjetas de vocabulario - flashcards —, etc.) o pidiendo ayuda (traducción, paráfrasis, oración que la incluya) a otra persona (el profesor, un compañero) — las segundas son estrategias sociales-. Y (2) las estrategias de consolidación del significado, que a su vez pueden ser sociales (estudiar y practicar el significado en grupo, con el profesor o interactuar con hablantes nativos), de memorización (asociar el significado de la palabra con una imagen, relacionar la palabra con una experiencia personal, con sinónimos y antónimos, crear mapas semánticos, agrupar palabras, utilizarlas en una oración, estudiar la ortografía y el sonido, decirlas en voz alta, parafrasear el significado, estudiarlas en una frase hecha, etc.), cognitivas (repetir la palabra de forma oral o escrita, tomar apuntes en clase, confeccionar listas o tarjetas de palabras, utilizar un cuaderno de vocabulario, etc.) y metacognitivas (autoevaluarse, estudiar varias veces la palabra, repasar, omitir la palabra desconocida, etc.).

Ahora bien, cabe preguntarse si existe alguna relación entre el uso de estas estrategias relacionadas con el aprendizaje de vocabulario y la variable sexo. En este sentido, Jiménez Catalán (2003:61-63) concluye que hombres y mujeres recurren prácticamente al mismo número de estrategias (si bien, el promedio en las mujeres es algo mayor y la diferencia es significativa) y, además, coinciden en el uso de las diez estrategias más y menos frecuentes. De las estrategias de descubrimiento, las más comunes son consultar el diccionario bilingüe, inferir significados por el contexto y preguntar al profesor o a los compañeros; y de las de consolidación: tomar apuntes en clase, asociar la palabra con un cognado de la L1, utilizar medios audiovisuales (escuchar canciones, ver películas, las noticias, etc.) y repetir la pala- 
bra en voz alta. La diferencia está en que las mujeres manejan más tipos de estrategias que los hombres, sobre todo cuando son estrategias de consolidación: de las 46 propuestas, las mujeres dicen emplear 31 y, en cambio, los hombres, solo 15. En las estrategias de descubrimiento, la diferencia es menor: de 14, ellas usan 9 y ellos, 5 (Jiménez Catalán, 2003:62).

En otro estudio, Moiinvaziri y Sahragard (2013:10-12) detectan que en el número de estrategias no difieren hombres y mujeres; pero sí que hay una diferencia significativa en dos casos concretos: ellas utilizan más la estrategia de memorización por medio del ensayo (repetición) y ellos se valen más de estrategias de activación.

En nuestro trabajo la propia mecánica de las tareas de entrenamiento conseguirá que los sujetos pongan en práctica diferentes estrategias de aprendizaje de vocabulario. Siguiendo la clasificación de Schmitt (1997), emplearán estrategias para descubrir el significado de una palabra por sí mismos (1) cuando las busquen en el diccionario monolingüe, que contiene una definición o un sinónimo; (2) si tratan de deducir el significado de las unidades léxicas por el contexto en el que están insertas; y también estrategias para consolidar el significado, ya sean de memorización: (3) al incluir la palabra en una oración que responda a la pregunta que les planteamos; cognitivas: (4) cuando la escriban al lado de la definición o del ejemplo correctos; y metacognitivas: (5) al practicar y repasar varias veces el significado de las palabras estímulo. Sin duda, habrá alumnos que utilicen más estrategias que los ayuden a descubrir y retener los significados, dependiendo de su propia experiencia como estudiantes, de sus habilidades y destrezas; pero, en cualquier caso, algunas estrategias son de uso obligado, lo que nos permite analizar si existe relación entre ellas y la variable sexo.

Como hemos dicho antes, gran parte de las investigaciones en el terreno del léxico estudia la eficacia de las técnicas de aprendizaje. En este sentido, las tareas productivas destacan como las más útiles en muchos de estos trabajos: Agustín Llach (2009), Browne (2003), Hulstijn y Laufer (2001), Keating (2008), Kim (2008), Kondo (2007), Laufer (2001; 2003), Pichette, De Serres y Lafontaine (2012), San-Mateo-Valdehíta (2012) y Webb (2005; 2009), entre otros. Si bien, hay investigadores, como Barcroft (2004) y Folse (2006), que llegan a conclusiones diferentes; para Barcroft (2004:329), la actividad productiva dificulta el aprendizaje de los nuevos vocablos, ya que consume recursos que sería posible emplear para procesar la forma de la palabra.

En este experimento emplearemos dos actividades de reconocimiento: (1) la selección de la definición correspondiente a la palabra estímulo y (2) la selección del ejemplo que contiene un equivalente semántico de dicha palabra; y (3) una de producción, que consiste en responder a una pregunta con una oración completa que incluya la palabra objeto de estudio y en la que quede claro que se conoce su significado.

Las tres tareas se centran en la palabra (form-focused activities). Frente a ellas, están las actividades centradas en el uso real de la lengua — es decir, en el sentido de los enunciados encaminados a lograr un objetivo de comunicación-, que dirigen la atención del aprendiz al contenido mismo del mensaje — son las llamadas focus on meaning activities-, (como la lectura extensiva, p. ej.). Y, puesto que la unidad léxica no es un medio para realizar una actividad comunicativa o de lengua auténtica [en cuyo caso sería una tarea con la atención hacia la forma — Focus on Form (FonF)—, sino que se manipulan secuencias aisladas, podemos 
clasificarlas como actividades centradas en las formas [Focus on FormS (FonFs)]. Tanto los ejercicios centrados en la forma como los centrados en las formas resultan ser muy eficaces para el aprendizaje de nuevos vocablos en L2 (Laufer, 2006:162); sin olvidar que deben ser complementarios al uso real de la lengua.

Las tres actividades que ponemos en práctica en este trabajo le exigen al aprendiz un grado de esfuerzo mental y de profundidad de análisis diferente (nivel de participación). Si utilizamos la escala propuesta por Hulstijn y Laufer (2001), de menor a mayor esfuerzo, se ordenan así: (1) la selección de definiciones requiere menos esfuerzo que (2) la selección de ejemplos que contienen un equivalente semántico de la palabra estímulo, y menos aún que (3) la redacción de oraciones. Los resultados obtenidos, teniendo en cuenta el entrenamiento, corroboran la hipótesis del nivel de participación (involvement load hypothesis), de Hulstijn y Laufer (2001:539-558), según la cual, mayor esfuerzo conlleva mayor retención: la escritura es la actividad que permite al aprendiz recordar más palabras (San-Mateo-Valdehíta, 2012: 243-247; 2013:27-28). Ahora en este estudio lo que haremos será aislar la variable sexo para analizar si alguna de las tareas facilita el aprendizaje de nuevas unidades léxicas a los hombres o a las mujeres.

A continuación, dedicaremos una sección a presentar los objetivos y las preguntas de investigación (§ 3). En el siguiente apartado, explicaremos la metodología: descripción de los participantes $(\$ 4.1)$, de las variables de estudio ( $\$ 4.2$ ), de los materiales de trabajo ( $\S$ 4.3 ), del procedimiento ( $\$ 4.4)$ y de los criterios de corrección del postest ( $\$ 4.5$ ). El quinto apartado está dedicado a la presentación de los resultados ( $\$ 5)$. Por último, expondremos el análisis de estos y las conclusiones $(\S 6)$ que se desprenden de la investigación.

\section{OBJETIVOS}

En este experimento hemos recogido información sobre una serie de variables personales y socioculturales del sujeto (el sexo, la procedencia escolar y el nivel sociocultural; la motivación, los años de estudio y el contexto de aprendizaje de la lengua meta, y el dominio de otras lenguas) — varias han sido estudiadas previamente en otras investigaciones (Matanzo Vicens, 1991, 1999; Reyes Díaz, 1995; San-Mateo-Valdehíta, 2005)— para comprobar si ejercen alguna influencia en el proceso de aprendizaje de vocabulario en L2. En San-Mateo-Valdehíta (2013-2014) recogemos las conclusiones obtenidas tras el análisis de algunas de estas variables: al igual que en los estudios anteriores, no parecen ser determinantes.

Ahora bien, en este trabajo, nos proponemos estudiar si el tipo de tarea empleado para aprender nuevas palabras en la L2 (español, en este caso) produce resultados significativamente diferentes entre los alumnos y las alumnas; es decir, teniendo en cuenta la variable sexo. Por otra parte, analizaremos, por separado, si los hombres y las mujeres sacan mayor partido a las actividades de reconocimiento (selección de definiciones y selección de ejemplos) o a las de producción (escritura de oraciones), y son capaces de retener más palabras con alguna de ellas. El análisis nos proporcionará información sobre la preferencia de los sujetos a la hora de emplear las estrategias de aprendizaje. 
Por lo tanto, los objetivos de nuestro experimento son (1) estudiar si la eficacia de diferentes tipos de ejercicios empleados para aprender nuevas palabras está condicionada por el factor sexo, y (2) examinar los resultados obtenidos por hombres y mujeres con cada tipo de tarea para comprobar si alguna es más eficaz que las otras.

Las preguntas que intentaremos responder para conseguir estos objetivos son las siguientes:

1. Cuando el entrenamiento se realiza con una actividad de reconocimiento como es la selección de definiciones, ¿obtienen resultados diferentes los hombres y las mujeres?

2. Cuando el entrenamiento se realiza con una actividad de reconocimiento como es la selección de ejemplos, ¿obtienen resultados diferentes los hombres y las mujeres?

3. Cuando el entrenamiento se realiza con una actividad de producción como es la escritura de oraciones, ¿obtienen resultados diferentes los hombres y las mujeres?

4. ¿Incorporan los hombres más palabras al lexicón mental si practican con (1) la selección de definiciones, (2) de ejemplos o con (3) la redacción de oraciones?

5. ¿Incorporan las mujeres más palabras al lexicón mental si practican con (1) la selección de definiciones, (2) de ejemplos o con (3) la redacción de oraciones?

\section{METODOLOGÍA}

En este estudio se compara el efecto cognitivo a corto plazo de dos clases de actividades de aprendizaje de vocabulario que se utilizan con frecuencia en L2: dos son de reconocimiento: (1) la selección de definiciones y (2) la selección de ejemplos; y una de producción: (3) la redacción de oraciones. Como veremos con más detalle en el apartado 4.4, dedicado a la explicación del procedimiento, los informantes se entrenan en una primera fase (pretest) con ejercicios de uno de esos tres tipos con el fin de aprender diez palabras estímulo, y después, en el postest, se comprueba el número de palabras que recuerdan. Los resultados obtenidos en el postest se examinan en relación con la variable sexo.

\subsection{Participantes}

Este estudio lo iniciaron 160 sujetos, pero diez fueron eliminados porque no completaron todas las actividades o no siguieron las instrucciones; de manera que, finalmente, la muestra quedó constituida por 150 estudiantes de español como L2, hablantes de inglés, de entre 19 y 21 años, y procedentes de la Universidad de Vanderbilt (Nashville, Tennessee, Estados Unidos), en la que cursan su tercer o cuarto año.

En el momento de realizar el experimento participan en un programa de estudios en el extranjero, concretamente en Madrid, donde pasan un trimestre académico. Uno de los requisitos del programa es haber aprobado determinadas asignaturas en su universidad y contar 
con un nivel intermedio de español [nivel B1, según el Marco Común Europeo de Referencia para las lenguas: aprendizaje, enseñanza y evaluación (MCER) (Consejo de Europa, 2002)]. De ahí que no consideráramos necesario aplicar ninguna otra media para estimar el nivel de conocimiento de la L2. El hecho de que los participantes tengan un nivel intermedio de español nos asegura que todos pueden comprender las instrucciones y el material utilizado en el estudio.

Las variables L1, edad y nivel de L2 quedan neutralizadas y no son objeto de estudio. Estamos ante una muestra muy homogénea desde este punto de vista.

\subsection{Variables de estudio}

El número de palabras que los participantes consiguen retener en la memoria y que queda reflejado en el postest que realizan tras el entrenamiento es la variable dependiente de la investigación. Y la variable independiente es el tipo de tarea de entrenamiento, que es (1) la selección de definiciones, (2) la selección de ejemplos o (3) la redacción de oraciones.

Los 150 alumnos son distribuidos de manera aleatoria en tres grupos de trabajo según el entrenamiento asignado: 47 sujetos practican con las definiciones; 51, con los ejemplos, y, 52 , con las oraciones (véase tabla 1).

Tabla 1. Distribución del grupo según las variables sexo y tarea de entrenamiento

\begin{tabular}{|c|c|c|c|}
\hline & $\mathbf{n}$ & Hombres & Mujeres \\
\hline Definiciones & $47(31,3 \%)$ & 6 & 41 \\
\hline Ejemplos & $51(34,0 \%)$ & 9 & 42 \\
\hline Oraciones & $52(34,7 \%)$ & 18 & 34 \\
\hline Total & 150 & $33(22 \%)$ & 117 (78\%) \\
\hline
\end{tabular}

Según la variable sexo, la muestra está formada por 117 mujeres y 33 hombres; es decir, el $78 \%$ son mujeres y el $22 \%$ son hombres (véase tabla 1). Esta diferencia entre hombres y mujeres es un claro reflejo de la realidad que encontramos en las aulas de L2 y es la consecuencia de no haber seleccionado previamente a los participantes del experimento. Estos forman parte de un grupo ya definido, que es la clase de español a la que asisten. Lo ideal sería que el tamaño de la muestra fuera similar en ambos grupos; aquí, al existir esta diferencia, no será posible considerar generales los resultados obtenidos. 


\subsection{Materiales}

Los materiales ${ }^{2}$ empleados en la sesión de trabajo son: un cuestionario, un diccionario, un pretest y un postest. En el cuestionario se recopila la información personal de los sujetos necesaria para analizar la muestra (véase en San-Mateo-Valdehíta y Andión Herrero, 2012: 90): el sexo, la edad, la L1, si han estudiado en centros públicos o privados, la profesión y formación de sus padres, los años que llevan estudiando español, dónde lo han estudiado y cuál es su motivación para hacerlo, etc. Para asegurar la confidencialidad de los datos aportados y no incurrir en daño moral alguno, dichos cuestionarios son anónimos; a cada sujeto se le asigna un código que, a la hora de analizar los resultados obtenidos en el postest, permite identificarlo.

El diccionario — procedente del estudio de Matanzo Vicens (1991) - consta de 20 palabras (ordenadas alfabéticamente): diez nombres, cinco adjetivos, cuatro verbos y un adverbio; todas de seis letras y sin tilde, para evitar la variación fonológica no controlada. Las palabras estímulo son diez pseudopalabras, con lo que descartamos la posibilidad de que algún informante las conozca y eliminamos toda posible transferencia de otras formas de cualquier lengua; cuatro son llanas y seis, agudas: barmil, casdel, cienge, leiver, sostro, tarjal, teifor, trelfa, trilen y zulvar. Las otras diez (seis llanas y cuatro agudas) son palabras de baja frecuencia ${ }^{3}$ : baltra, fauces, filial, juglar, lacrar, nuncio, plagar, plagio, ristra y viable. En el diccionario se indica, al lado de cada palabra, la categoría gramatical y un sinónimo o una definición en la L2 — que ha sido adaptada al nivel de los alumnos—, como vemos en los siguientes ejemplos:

Baltra (n.): Vientre, panza.

Cienge (n.): Conjunto de personas, animales o cosas; multitud, gran cantidad.

Fauces (n.): Parte posterior de la boca de los mamíferos.

Filial (adj.): Perteneciente al hijo.

Teifor (adj.): Fundamental.

Zulvar (v.): Forzar a una persona a obrar de manera determinada.

Las tareas del pretest y del postest son similares. En las de reconocimiento, los sujetos deben escribir la palabra estímulo al lado de la definición o del ejemplo correspondiente, como vemos en los dos ejemplos siguientes:

${ }^{2}$ Seleccionados y adaptados del trabajo de Matanzo Vicens (1991:94-111), que realizó en el ámbito de la L1 (español), del cual nuestro experimento es, en parte, una réplica. Y, en el estudio piloto (San-Mateo-Valdehíta, 2005:106; 109113; San-Mateo-Valdehíta y Andión Herrero, 2012:79-81), se determinó que eran los adecuados para recoger los datos.

${ }^{3} \mathrm{La}$ frecuencia no es la misma en todos los casos, hay algunas palabras menos frecuentes que otras. En la lista de frecuencias del Corpus de referencia del español actual (CREA) (http://corpus.rae.es/lfrecuencias.html), que recoge más de ciento sesenta millones de formas, baltra aparece en la posición 162 982, lo que demuestra su bajo índice de uso; lacrar (en concreto, lacrado) ocupa la posición 64 060; ristra, la 50 551; y juglar, la 49180 (y juglares, la 44 109). Alrededor de la posición 25000 se encuentran: fauces (en la 25 345), plagio (en la 23 360), plagar (plagado, en la 23 317) y nuncio (en la 21 805). Sin embargo, hay dos palabras del diccionario que son mucho más frecuentes: filial, que aparece en la posición 7542 y viable, en la 9675. 
Escribe la palabra ZULVAR al lado de la definición que le corresponda:

1. Prevenir, precaver.

2. Dañar la salud de una persona, contagiarle una enfermedad

3. Forzar a una persona a obrar de manera determinada.

4. Llenar o cubrir a alguna persona o cosa de algo nocivo o no conveniente.

Escribe la palabra ZULVAR al lado del ejemplo que le corresponda:

1. El estudiante no cumplió con las normas del curso.

2. Después del accidente el hombre quedó físicamente irreconocible.

3. Trató inútilmente de evitar el accidente.

4. Unos jóvenes obligaron a Luis a cometer un delito grave.

En el ejercicio de producción, se trata de responder a la pregunta planteada con una oración completa que incluya la palabra estímulo y que demuestre que se conoce su significado. Ejemplo:

Contesta a la siguiente pregunta con una oración completa. Utiliza la palabra indicada EN MAYÚSCULAS y negrita y subráyala. Tu contestación debe demostrar que conoces el significado de dicha palabra: ¿Ha tratado alguien de ZULVARTE a cometer algún delito grave? Explícalo.

Del pretest, que consta de diez preguntas, hay tres modelos: en cada uno de ellos se trabaja con un tipo de tarea: (1) selección de definiciones, (2) selección de ejemplos, y (3) redacción de oraciones. El postest, en cambio, es para todos los participantes igual: tiene tres secciones (una por tipo de ejercicio), con diez preguntas cada una; es decir, en total, consta de treinta ítems.

\subsection{Procedimiento}

Los datos se recogen de nueve grupos de alumnos de la clase de la asignatura Gramática española del curso intensivo al que asisten los participantes a su llegada a España. Los ejercicios del estudio se plantean como una actividad más de clase.

Al inicio de la sesión de trabajo, antes de pedirles que completen el cuestionario con la información personal, se les explica que van a participar en una investigación relacionada con el aprendizaje y enseñanza del español como L2. Una vez finalizada, se les revela el propósito concreto del estudio - no se hace al inicio, pues podía desvirtuar o sesgar los resultados obtenidos- . Así que, en principio, no intentarán deliberadamente retener las palabras estímulo con las que van a trabajar, por lo que se trata de aprendizaje no intencional.

La mecánica del experimento es la siguiente. Primero, se divide a los alumnos, aleatoriamente, en tres grupos, y cada uno de ellos, en el pretest, trabajará con una actividad: el Grupo 1 con la selección de definiciones; el 2, con la selección de ejemplos y el 3, con la escritura 
de oraciones. Después se reparte el diccionario y la investigadora lee en voz alta las palabras, junto con sus definiciones correspondientes. A continuación, comienza la fase de entrenamiento, que se compone de dos series de actividades: en la primera, los sujetos pueden consultar el diccionario; en la segunda, no. En ambas, disponen de 45 segundos para completar cada uno de los diez ítems; y, transcurrido ese tiempo, se indica cuál es la definición correcta de la palabra con la que se está trabajando — esta es la retroalimentación que reciben-. Finalmente, durante 15 minutos se realiza el postest, donde los sujetos deben demostrar su conocimiento de las diez palabras estímulo en los ejercicios de reconocimiento (selección de definiciones y de ejemplos) y en el de producción (escritura de oraciones).

\subsection{Criterios de corrección del postest}

La corrección del postest se ha efectuado de la siguiente manera: cada ítem correcto cuenta un punto; el incorrecto o el no contestado, cero. Seguimos el sistema de puntuación habitual en otros experimentos similares [Hulstijn y Laufer (2001); Laufer (2001, 2003)] y no hacemos distinción entre dejar la pregunta en blanco y responderla incorrectamente, ya que es una evaluación de resultados y no del proceso de aprendizaje.

En el caso de las tareas de selección solo una de las respuestas es correcta, por lo que la corrección es automática. Para el ejercicio de redacción, hemos establecido una serie de criterios basados en la experiencia del estudio piloto (San-Mateo-Valdehíta, 2005), con el fin de que la corrección sea lo más objetiva posible, ya que se trata de interpretar si en la oración dada como respuesta queda demostrado que el sujeto conoce el significado de la palabra estímulo. En esta corrección, los errores ortográficos y sintácticos, como, por ejemplo, los de esta respuesta: «Porque tengo morales ciertos, no permito que alguien me zulvaría a cometer algún delito grave» (51_E), no se tienen en cuenta ni penalizan de manera alguna. Y, aunque en las instrucciones se pide explícitamente que se incluya la palabra estímulo en la respuesta, se acepta si se utiliza un sinónimo de esta. Entendemos que esto está justificado porque en la pregunta se inserta el estímulo, de manera que no incluirlo en la respuesta es, en parte, el comportamiento habitual de los hablantes nativos, en su afán por evitar la repetición. P. ej., a la pregunta: «¿Ha tratado alguien de ZULVARTE a cometer algún delito grave? Explícalo», un sujeto responde: «Sí, tuve un enemigo que me obligó a hacer trampas y completar la tarea suya» (9_D) —se usa obligar por zulvar-.

\section{RESULTADOS}

El número de palabras que cada sujeto es capaz de recordar, es decir, el resultado obtenido en el postest, ha sido introducido en el programa SPSS-Statistical Package for the Social Sciences (IBM SPSS Statistics 19) para realizar el análisis estadístico descriptivo y hallar las medias aritméticas, la desviación típica o estándar y el error típico. El promedio de aciertos se ha calculado por alumno, según la actividad de entrenamiento y teniendo en cuenta la variable sexo. Después, con el objetivo de comprobar si la diferencia entre estos promedios es significativa, hemos aplicado las pruebas estadísticas pertinentes, en concreto la prueba T de Student y el análisis de varianza (ANOVA). 
El primer dato que obtenemos es el promedio total de aciertos en el postest —es decir, en número de palabras que los participantes recuerdan - y comprobamos que es una cifra elevada: 8,16 sobre 10 [ $\mathrm{n}=150$; desviación estándar (a partir de ahora, DE): 1,64; error típico (ET): 1,34]. Esto quiere decir que los dos tipos de tareas de entrenamiento son eficaces para ayudar al aprendiz de L2 a incorporar nuevas palabras al lexicón mental (San-Mateo-Valdehíta, 2012:235; 2013:29).

Por otro lado, se confirma que las actividades de reconocimiento son menos eficaces que la de producción: en el grupo que se ha entrenado con la selección de definiciones, el promedio de aciertos es 7,54, sobre un máximo de 10 ( $n=47$; DE: 2,34; ET: 0,2); y en el grupo de selección de ejemplos, el promedio es 8,10 ( $\mathrm{n}=51$; DE: 2,2; ET: 0,18). Y, por último, en el grupo entrenado con la tarea de producción, el promedio es 8,77 ( $\mathrm{n}=52$; DE: 1,46; ET: 0,12 ). La diferencia entre el primer y tercer grupo es significativa con un nivel de confianza del 95\%, según el análisis de varianza $[\mathrm{F}(2,147)=7,631 \mathrm{p}=0,001]$ y las pruebas post hoc (Scheffé) (p = 0,001) (San-Mateo-Valdehíta, 2012: 243-247; 2013: 28); es decir, que el tipo de entrenamiento conlleva resultados significativamente diferentes, desde el punto de vista estadístico, y la redacción de oraciones es más eficaz que el trabajo con definiciones.

Ahora bien, nuestro objetivo aquí es estudiar la posible relación entre la tarea de aprendizaje y la variable sexo; así que, a continuación, presentamos los promedios de las puntuaciones obtenidas aislando esta variable. Y, dentro de cada subgrupo (hombres, por un lado, y mujeres, por otro), analizaremos la incidencia de las actividades de entrenamiento: (1) la selección de definiciones, (2) la selección de ejemplos y (3) la redacción de oraciones.

En primer lugar, teniendo en cuenta los resultados del postest del grupo completo, sin diferenciar según la tarea de entrenamiento, el promedio de aciertos conseguidos por hombres y mujeres apenas se diferencia en una centésima: $8,16(n=33$; DE: 1,56 ; ET: 0,27) y 8,15 ( $\mathrm{n}=117$; DE: 1,67; ET: 0,15), respectivamente (véase tabla 2); por lo que la variable sexo no parece distinguir la incorporación de palabras al lexicón mental. Y así lo confirma la T de Student, según la cual la probabilidad de error es superior a 0,05: $\mathrm{t}(148)=0,024 \mathrm{p}=0,981$. Por lo tanto, con un nivel de confianza del 95\%, la variable sexo no es un factor que influya en la incorporación de palabras al lexicón mental (San-Mateo-Valdehíta, 2013-2014: 262).

Tabla 2. Resumen del promedio de aciertos según las variables sexo y tarea de entrenamiento

\begin{tabular}{|l|c|c|c|c|c|c|c|c|}
\hline & \multicolumn{3}{|c|}{ Hombres } & \multicolumn{4}{c|}{ Mujeres } \\
\cline { 2 - 10 } & $\mathbf{n}$ & Promedio & DE & ET & $\mathbf{n}$ & Promedio & DE & ET \\
\hline Definiciones & 6 & 7,33 & 2,11 & 0,86 & 41 & 7,57 & 1,96 & 0,31 \\
\hline Ejemplos & 9 & 7,74 & 1,69 & 0,56 & 42 & 8,17 & 1,63 & 0,25 \\
\hline Oraciones & 18 & 8,65 & 1,17 & 0,28 & 34 & 8,83 & 0,95 & 0,16 \\
\hline Total & 33 & 8,16 & 1,56 & 0,27 & 117 & 8,15 & 1,67 & 0,15 \\
\hline
\end{tabular}


En segundo lugar, si nos fijamos en los resultados de los hombres y de las mujeres dentro de cada uno de los tres grupos establecidos según el entrenamiento (véase tabla 2 y gráfico 1), comprobaremos que las mujeres han obtenido mejores resultados que los hombres independientemente del tipo de tarea con el que hayan practicado ${ }^{4}$.

\section{Gráfico 1. Promedio de aciertos según las variables sexo y tarea de entrenamiento}

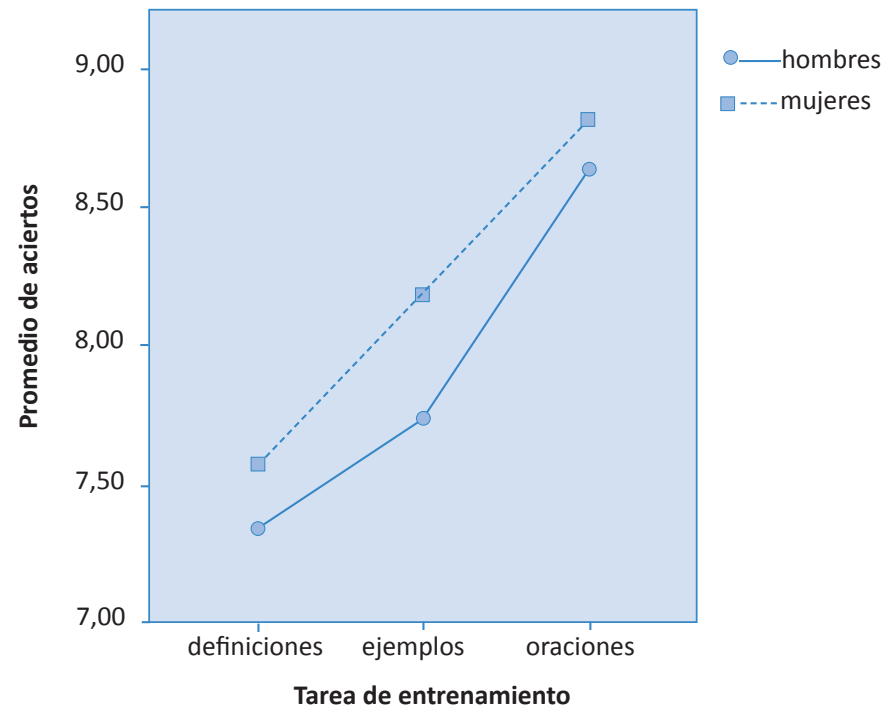

Cuando se trata de actividades de reconocimiento, las mujeres consiguen 7,57 aciertos frente a los 7,33 de los hombres — con el ejercicio de selección de definiciones- y 8,17 frente a 7,74 — con el de selección de ejemplos—. Mediante el análisis estadístico comparamos estas puntuaciones: la prueba $\mathrm{T}$ indica que la diferencia entre los promedios no es significativa con un nivel de confianza del $95 \%$, pues la probabilidad de error es superior a 0,05 : en el caso del grupo que ha trabajado con la selección de definiciones: $\mathrm{t}(45)=-0,273 \mathrm{p}=0,786 ; \mathrm{y}$ en el del grupo de selección de ejemplos: $\mathrm{t}(49)=-0,722 \mathrm{p}=0,474$.

Cuando se entrenan con la tarea de producción, también las mujeres obtienen la mayor puntuación: 8,83 frente a 8,65. Si bien, la diferencia no es significativa tampoco, como indica la prueba T: t $(50)=-0,615 \mathrm{p}=0,541$. En definitiva, la diferencia del número de palabras incorporadas al lexicón mental por los hombres y las mujeres no se puede justificar por la actividad de aprendizaje, sea de reconocimiento o de producción.

${ }^{4}$ Nótese que al hallar el promedio total de aciertos, por un lado, de los hombres y, por otro, el de las mujeres, la puntuación de ellos es una centésima mayor $(8,16)$ que la de las mujeres $(8,15)$. Esto sucede porque el número de individuos no es el mismo en cada uno de los grupos establecidos según la tarea de entrenamiento (véase tabla 2): el promedio global se calcula con el número total de participantes ( 33 hombres y 117 mujeres) y el promedio de cada grupo con el número de los sujetos pertenecientes a él (6, 9 y 18, en el caso de los hombres; y 41, 42 y 34, en el de las mujeres). 
La mejor puntuación tanto de los hombres como de las mujeres se ha alcanzado con el entrenamiento de la escritura de oraciones $(8,65$ y 8,83 aciertos), después con la selección de ejemplos (7,74 y 8,17 aciertos) y, finalmente, con la selección de definiciones $(7,33$ y 7,57 aciertos) (véase tabla 2); con lo cual, aquí también se confirma la hipótesis del nivel de participación de Hulstijn y Laufer (2001:543-545): todos recuerdan más palabras cuando han practicado con la actividad que requiere mayor esfuerzo mental.

En tercer lugar, analizaremos los resultados conseguidos por los hombres, por un lado, y las mujeres, por el otro, cuando se han entrenado con las tareas de reconocimiento y con la de producción para comprobar si con alguna de ellas incorporan un mayor número de palabras en el lexicón mental. Para ello, comparamos los promedios obtenidos con cada una de las actividades de entrenamiento dentro de cada subgrupo.

En el grupo de los hombres, destacan los que se han entrenado con la redacción de oraciones $(8,65$ aciertos; $\mathrm{n}=18$; DE: 1,17 ; ET: 0,28$)$ y la media más baja es la de los que practicaron con la selección de definiciones (7,33 aciertos; $n=6$; DE: 2,11; ET: 0,86); en la posición intermedia, quedan los que seleccionaron ejemplos $(7,74$ aciertos; $n=9$; DE: 1,69; ET: 0,56) (véase tabla 2). Sin embargo, el análisis estadístico indica que la diferencia entre estos promedios no es significativa en un nivel de confianza del $95 \%$, pues la probabilidad de F de Snedecor es superior a 0,05: F $(2,30)=2,191 \mathrm{p}=0,129$ (véase tabla 3). Esto significa que el tipo de tarea de entrenamiento no puede ser considerado la causa de dicha diferencia.

Tabla 3. Resultados del ANOVA para las variables sexo: hombre y tarea de entrenamiento

\begin{tabular}{|l|c|c|c|c|c|}
\hline \multicolumn{7}{|c|}{ ANOVA $^{-2}$} \\
\hline Suma de cuadrados & gl & $\begin{array}{c}\text { Media } \\
\text { cuadrática }\end{array}$ & F & Sig. \\
\hline Inter-grupos & 9,971 & 2 & 4,986 & 2,191 &, 129 \\
\hline Intra-grupos & 68,278 & 30 & 2,276 & & \\
\hline Total & 78,249 & 32 & & & \\
\hline a. sexo = hombre & & & & \\
\hline
\end{tabular}

En el grupo de mujeres, las que han practicado con la redacción son las que más palabras retienen: 8,83 ( $\mathrm{n}=34$; DE: 0,95; ET: 0,16); después, las que se han entrenado con la selección de ejemplos (8,17 aciertos; $\mathrm{n}=42$; DE: 1,63; ET: 0,25), y, por último, las del subgrupo de selección de definiciones (7,57 aciertos; $n=41$; DE: 1,96; ET: 0,31) (véase tabla 2). En este caso, la diferencia entre los promedios sí que es significativa (véase tabla 4), ya que la probabilidad de F de Snedecor es inferior a 0,05: F $(2,114)=5,806$ p = 0,004. 
Tabla 4. Resultados del ANOVA para las variables sexo: mujer y tarea de entrenamiento

\begin{tabular}{|c|c|c|c|c|c|}
\hline \multicolumn{2}{|c|}{ ANOVA $^{\mathbf{2}}$} \\
& Suma de cuadrados & gl & $\begin{array}{c}\text { Media cuadrá- } \\
\text { tica }\end{array}$ & F & Sig. \\
\hline Inter-grupos & 29,735 & 2 & 14,867 & 5,806 &, 004 \\
\hline Intra-grupos & 291,940 & 114 & 2,561 & & \\
\hline Total & 321,675 & 116 & & & \\
\hline
\end{tabular}

a. sexo $=$ mujer

Los procedimientos de comparaciones múltiples post hoc determinan entre qué pares la diferencia es significativa (véanse los resultados de la prueba de Scheffé en la tabla 5). De esta manera, comprobamos que la diferencia entre la puntuación de las mujeres que trabajan con la redacción de oraciones y la de las que lo hacen con la selección de definiciones es significativa con un nivel de confianza del 95\% ( $\mathrm{p}=0,004$, en la tabla 5 se señala con un asterisco; las pruebas de Tukey y de Bonferroni elevan el nivel de confianza un punto: $p=0,003$ ). Esto quiere decir que, entre las mujeres, la actividad de producción — la escritura- es significativamente más eficaz que la de reconocimiento consistente en seleccionar la definición correspondiente a la palabra estímulo.

Tabla 5. Resultados de la prueba de Scheffé para las variables sexo: mujer y tarea de entrenamiento

\begin{tabular}{|c|c|c|c|c|c|c|}
\hline \multicolumn{7}{|c|}{ Comparaciones múltiples ${ }^{\mathrm{a}}$} \\
\hline \multirow{2}{*}{ (I) actividad } & \multirow{2}{*}{ (J) actividad } & \multirow{2}{*}{$\begin{array}{l}\text { Diferencia de } \\
\text { medias (I-J) }\end{array}$} & \multirow{2}{*}{ Error típico } & \multirow{2}{*}{ Sig. } & \multicolumn{2}{|c|}{ Intervalo de confianza al 95\% } \\
\hline & & & & & Límite inferior & Límite superior \\
\hline \multirow{2}{*}{ oraciones } & ejemplos & 65873 & ,36918 & ,208 &,- 2569 & 1,5744 \\
\hline & definiciones & $1,26423 *$ & 37119 & ,004 & ,3436 & 2,1849 \\
\hline \multirow{2}{*}{ ejemplos } & oraciones &,- 65873 & ,36918 & ,208 & $-1,5744$ & 2569 \\
\hline & definiciones & ,60550 & ,35133 & ,231 &,- 2659 & 1,4769 \\
\hline \multirow{2}{*}{ definiciones } & oraciones & $-1,26423^{*}$ & ,37119 & ,004 & $-2,1849$ &,- 3436 \\
\hline & ejemplos &,- 60550 & ,35133 & ,231 & $-1,4769$ & 2659 \\
\hline
\end{tabular}




\section{ANÁLISIS Y CONCLUSIONES}

Los resultados de nuestro estudio sobre la posible influencia de la variable sexo en el aprendizaje de vocabulario por parte del estudiante de español como L2 están en consonancia con los obtenidos en el estudio piloto, donde concluimos que «no existe relación entre el sexo y la velocidad de incorporación de nuevas palabras y, efectivamente, la diferencia, con un nivel de confianza al 95\%, no ha sido significativa» (San-Mateo-Valdehíta, 2005:84). Las similitudes entre hombres y mujeres puede que sean más que las diferencias, debido al papel homogeneizador de las aulas de L2:

As human beings, males and females are more alike than different; although there may be sex differences in language learning due to innate and social causes, research carried out so far is nor conclusive enough to determine absolutely different ways of learning for the two sexes; to this should be added the role of encouraging uniformity played by educational systems and the similarity of foreign language classes (Jiménez Catalán, 2003:64).

En este caso, además, no hay que olvidar que los participantes proceden de la misma universidad, aunque no todos cursan la misma carrera; y han estudiado la L2 en las mismas aulas, lo cual podría influir incluso en los hábitos de estudio.

En experimentos en los que se trabaja con la misma tipología de ejercicios, pero en el campo de la L1, como el llevado a cabo por Matanzo Vicens (1991) en Puerto Rico, la variable sexo tampoco resulta ser determinante en el aprendizaje de vocabulario (Matanzo Vicens, 1991:56-77) — aunque la autora no aporta análisis estadístico—. Sin embargo, en el estudio de Reyes Díaz (1995), realizado en Las Palmas de Gran Canaria, también en L1, sí que lo es teniendo en cuenta los datos globales (de los 254 informantes) y son las mujeres las que mejor puntuación consiguen (1995:163-164); pero la diferencia deja de ser significativa en el grupo que cursa COU (Curso de Orientación Universitaria), cuyos alumnos tienen una media de 17 años —en ese experimento participan otros dos grupos de informantes: estudiantes de $1 .^{\circ}$ de BUP (Bachillerato Unificado Polivalente), con 14 años de media, y de $7 .^{\circ}$ de EGB (Educación General Básica), con 12 años-. La diferencia entre las puntuaciones obtenidas por hombres y mujeres (Reyes Díaz, 1995:169; 172; 174) se va reduciendo progresivamente a medida que los sujetos cumplen años y avanzan en el sistema educativo, hasta que llega a no ser significativa - justo cuando llegan a la edad que tienen los informantes del experimento de Puerto Rico-; los alumnos de nuestro estudio han rebasado esa edad, pues tienen entre 19 y 21 años. Parece que la diferencia desaparece cuando termina la adolescencia.

En realidad, muchas de las investigaciones sobre el desarrollo del lexicón mental de los aprendices de L2 se centran en la efectividad de la actividades de instrucción, más que en las variables personales que pudieran influir en él, con lo cual la comparación de resultados de una variable como el sexo en la práctica no es viable.

En nuestro estudio hemos analizado precisamente la eficacia de dos tipos de tareas: de reconocimiento y de producción, teniendo en cuenta dicha variable personal. Hemos partido del hecho de que el ejercicio de entrenamiento sí que produce resultados diferentes y significativos, siendo más eficaz el que obliga al sujeto a producir (la redacción de oraciones) que los que requieren solamente reconocer la definición o el ejemplo correctos (San-Mateo-Valdehíta, 2013:27-29). Cuando hemos dividido la muestra teniendo en cuenta 
la variable sexo, hemos comprobado que los resultados van en la misma dirección: tanto los hombres como las mujeres son capaces de retener más palabras cuando se entrenan con la actividad de producción; en cambio, recuerdan menos cuando trabajan con la de seleccionar la definición correcta.

$\mathrm{Al}$ analizar por separado cada uno de los tres grupos: (1) el que se ha entrenado con la actividad de seleccionar de definiciones, (2) el que ha practicado con la selección de ejemplos y (3) el que ha contestado a la pregunta redactando una oración que incluye la palabra estímulo, hemos comprobado que los resultados logrados por los hombres y por las mujeres no son significativamente diferentes entre sí. El sexo, en ese sentido, no es un factor diferenciador — lo cual responde a las tres primeras preguntas que nos planteábamos en $\S 3$ -

Para contestar a la cuarta y quinta cuestión sobre si ellos o ellas retienen más palabras cuando practican con un tipo concreto de ejercicio, hemos analizado los resultados alcanzados por los hombres, por un lado, y por las mujeres, por el otro, cuando han llevado a cabo las diferentes tareas. La conclusión es que ellos no muestran especial predilección por ninguna de las tres actividades; en cambio, las mujeres, cuando practican con la actividad de elaboración, consiguen resultados significativamente mejores que con el mero reconocimiento de las definiciones; es decir, la escritura las ayuda a retener un mayor número de palabras.

Esto puede explicarse por el tipo de estrategias que utilizan frecuentemente las mujeres, tal y como otros estudios confirman. Así, en el de Jiménez Catalán (2003:62; 66) las mujeres se decantan más que los hombres, sobre todo, por estrategias de consolidación y, entre ellas están las estrategias de memorización, como lo es la escritura de oraciones con la palabra estímulo. Las estrategias de descubrimiento, como buscar el significado en el diccionario o deducirlo por el contexto — que también se ponen en práctica con las tareas de entrenamiento empleadas aquí- están encaminadas simplemente a solucionar el problema de comprensión (Jiménez Catalán, 2003:63), pero no suponen mayor esfuerzo para que la nueva unidad léxica pase a la memoria a largo plazo; de manera que resultan menos eficaces en sí mismas si no van acompañadas de otra estrategia de consolidación. Quizá se trate de una diferencia que vaya más allá de la predilección por una determinada actividad o el desarrollo de una estrategia en concreto y tenga que ver con los estilos de aprendizaje.

La importancia del estudio de las estrategias de aprendizaje reside en la posibilidad de enseñarlas explícitamente en el aula de L2 y que así el alumno se entrene con ellas para aplicarlas de forma eficaz, y consiga, entre otras cosas, aprender gran cantidad de unidades léxicas por sí mismo. Higueras García (2004:20-22) resalta la relevancia de entrenar a los aprendices en dos de ellas, que, no por casualidad, son dos de las más utilizadas: (1) el manejo del diccionario y, sobre todo, cuándo conviene consultarlo; y (2) la deducción del significado por el contexto (Nation, 2001).

En nuestro estudio ha quedado manifiesto que sí hay una actividad más eficaz que otra y que la estrategia que se practica con ella es especialmente útil en el caso de las mujeres. En este sentido, los resultados aportan información sobre con qué tarea se retienen más vocablos y sobre las estrategias de aprendizaje a las que recurren los aprendices de español como L2 de cada sexo, y están en consonancia con el trabajo de Jiménez Catalán (2003). Sin embargo, conviene ser conscientes de que no parece didácticamente plausible enseñar en el aula a ellos 
unas estrategias y a ellas, otras; más bien el docente debe intentar que el número y tipo de estrategias empleadas aumente con el fin de que la ampliación del lexicón mental se produzca de forma más eficiente.

Finalmente, cabe señalar algunas limitaciones de este estudio. Teniendo en cuenta la diferencia muestral entre los dos grupos de informantes que establece la variable sexo, precisaría ser replicado con un mayor número de varones para refrendar las conclusiones, que, de momento, solo deben considerarse parciales, si bien están en la misma línea de otras investigaciones, como hemos apuntado antes. Además, pensamos que la ampliación de este experimento con una muestra más diversa y amplia de informantes, en cuando a la L1 y la L2, la procedencia, la edad y el nivel de L2, e incluyendo la variable estilos de aprendizaje, permitiría estudiar su posible interacción con las actividades y las estrategias de aprendizaje y la variable sexo, de manera que se superarían las limitaciones de este trabajo. Por el momento, las conclusiones no se deben extrapolar a grupos de sujetos con otras L1, edades y niveles de español como L2. Por otra parte, este estudio no nos permite establecer una correlación entre el empleo de una determinada tarea de entrenamiento y el éxito o fracaso en el aprendizaje de la L2 en general, ya que se trata de una investigación descriptiva; si bien, podríamos predecir que la práctica con las actividades más eficaces está relacionada con una mayor incorporación de palabras al lexicón mental, que redundará en un mayor dominio general de la L2.

\section{Agradecimientos}

Quiero agradecer a los evaluadores anónimos de la revista sus observaciones y comentarios sobre la primera versión del artículo.

\section{REFERENCIAS BIBLIOGRÁFICAS}

Agustín Llach, María Pilar (2009): «The effect of reading only, reading and comprehension, and sentence writing in lexical learning in a foreign language: some preliminary results», Revista Española de Lingüística Aplicada, 22, PÁGS. 9-33.

Arifuddin, Hasan, Susanto Susanto y Suharsono Suharsono (2013): «Typology of implicature questions in TOEFL-Like and causes of failure according to gender», International Journal of Linguistics, 5.6, págs. 37-74.

BACON, Susan M. (1992): «The relationship between gender, comprehension, processing strategies, and cognitive and affective response in foreign language listening», The Modern Language Journal, 76.2, págs. 160-178.

BARCROFT, JoE (2004): «Effects of sentence writing in second language lexical acquisition», Second Language Research, 20.4, págs. 303-334.

Bowden, Harriet Wood, Cristina Sanz y Catherine A. Stafford (2005): «Individual differences: Age, sex, working memory, and prior knowledge», Mind and context in adult second language acquisition: Methods, theory, and practice, págs. 105-140.

Browne, Charles (2003): Vocabulary acquisition through reading, writing and tasks: a comparison (tesis doctoral inédita), Temple University Japan.

Consejo de Europa (2002): Marco común europeo de referencia para las lenguas: aprendizaje, enseñanza y evaluación (MCER), Estrasburgo: http://www.cvc.cervantes.es/obref/marco/indice. htm (20-04-2015). 
FARHADY, HosseIN (1982): «Measures of language proficiency from the learner's perspective», TESOL quarterly, 16.1, págs. 43-59.

FoLSE, KeITH S. (2006): «The effect of type of written exercise on L2 vocabulary retention», TESOL Quarterly, 40.2, págs. 273-293.

Higueras García, Marta (2004): «Claves prácticas para la enseñanza del léxico», Carabela. La enseñanza de léxico en español como segunda lenguallengua extranjera, 56, págs. 5-25.

Hulstijn, Jan H. y Batia Laufer (2001): «Some empirical evidence for the Involvement Load Hypothesis in vocabulary acquisition», Language Learning, 51.3, págs. 539-558.

Jiménez Catalán, Rosa María (2002): «El concepto de competencia léxica en los estudios de aprendizaje y enseñanza de segundas lenguas», Atlantis, 24.2, págs. 149-162.

- (2003): «Sex differences in L2 vocabulary learning strategies», International Journal of Applied Linguistics, 13.1, págs. 54-77.

Keating, Gregory D. (2008): «Task effectiveness and word learning in a second language: the Involvement Load Hypothesis on trial», Language Teaching Research, 12.3, págs. 365-386.

KIM, YouJIN (2008): «The role of task-induced involvement and learner proficiency in L2 vocabulary acquisition», Language Learning, 58.2, págs. 285-325.

Kondo, Hiroyuki (2007): «The effects of semantic elaboration on L2 vocabulary learning», Research Journal of Jin-Ai University, 6, págs. 71-78.

LakofF, Robin Tolmach (1973): «Language and woman's place», Language in society, 2.1, págs. 45-80.

Larsen-Freeman, Diane y Michael H. Long (1994): Introducción al estudio de la adquisición de segundas lenguas, Madrid, Gredos.

LAUFER, BATIA (2001): «Reading, word-focused activities and incidental vocabulary acquisition in a second language», Prospect, 16.3, págs. 44-54.

- (2003): «Vocabulary acquisition in a second language: Do learners really acquire most vocabulary by reading? Some empirical evidence», The Canadian Modern Language Review, 59.4, págs. 567-587.

- (2006): «Comparing Focus on Form and Focus on Forms in second-language vocabulary learning», The Canadian Modern Language Review, 63.1, págs. 149-166.

MaiguashCa, Raffaella Uslenghi (1993): «Teaching and learning vocabulary in a second language: past, present, and future directions», The Canadian Modern Language Review, 50.1, págs. 83-100.

Matanzo Vicens, GloRia (1991): Vocabulario y enseñanza: Estudio de la relación existente entre los métodos empleados y la incorporación del léxico nuevo a la competencia lingüística de estudiantes universitarios puertorriqueños (tesis doctoral inédita), Universidad de Puerto Rico.

- (1999): «Incorporación léxica y metodología de enseñanza». En Samper Padilla, José Antonio y Magnolia Troya Déniz (coords.): Actas del XI Congreso Internacional de la Asociación de Lingüística y Filología de la América Latina. Las Palmas de Gran Canaria, del 22 al 27 de julio de 1996, Las Palmas de Gran Canaria, Universidad de Las Palmas de Gran Canaria, págs. 16231628.

MoiInVAZIRI, MARJAN y RAHMAN SAHRAGARD (2013): «English vocabulary learning of Iranian university students and its relation to age and gender», International Journal of Instructional Technology and Distance Learning, 10.7, págs. 3-18.

NATION, I. S. Paul (2001): Learning vocabulary in another language, Cambridge, CUP.

O'Malley, J. Michael y Anna Uhl Chамот (1990): Learning strategies in second language acquisition, Cambridge, CUP.

OXFORD, REBECCA (1990): Language learning strategies. What every teacher should know, Nueva York, Newbury House.

Oxford, Rebecca, Martha Nyikos y Madeline Ehrman (1988): «Vive la difference? Reflections on sex differences in use of language learning strategies», Foreign Language Annals, 21.4, págs. 321-329. 
Phaкiti, Aeк (2003): «A closer look at gender and strategy use in L2 reading», Language Learning, 53.4, págs. 649-702.

Pichette, Francois, Linda de Serres y Marc Lafontaine (2012): «Sentence reading and writing for second language vocabulary acquisition», Applied Linguistics, 33.1, págs. 66-82.

RADWAN, Adel ABu (2011): «Effects of L2 proficiency and gender on choice of language learning strategies by university students majoring in English», Asian EFL Journal, 13.1, págs. 114-162.

Real Academia Española: Banco de datos (CREA) [en línea]. Corpus de referencia del español actual: http://www.rae.es (20-04-2015).

Reyes Díaz, M. ${ }^{a}$ Josefa (1995): Enriquecimiento de la competencia léxica: análisis estadístico [Recurso electrónico] (tesis doctoral), Las Palmas de Gran Canaria, Universidad de Las Palmas de Gran Canaria.

SALEM, NAdA Michel (2006): The role of motivation, gender, and language learning strategies in EFL proficiency (tesis doctoral inédita), University of Beirut.

San-Mateo-Valdehíta, Alicia (2005): Aprendizaje de léxico en español como segunda lengua. Investigación sobre tres métodos (trabajo de investigación Diploma de Estudios Avanzados, UNED), Biblioteca redELE: http://www.mecd.gob.es/redele/Biblioteca-Virtual/2005/memoriaMaster/1-Semestre/SAN-MATEO-UNED.html.

- (2012): Aprendizaje de léxico en español como segunda lengua/lengua extranjera: investigación sobre la efectividad de tres tipos de actividades para aprender vocabulario (tesis doctoral inédita), UNED.

- (2013): «El efecto de tres actividades centradas en las formas (Focus on formS, FonFs): la selección de definiciones, la selección de ejemplos y la escritura de oraciones, en el aprendizaje de vocabulario en segundas lenguas», RacL-Revista Electrónica de Lingüística Aplicada, 12, págs. 17-36: http:// dialnet.unirioja.es/descarga/articulo/4648292.pdf (20-04-2015).

- (2013-2014): «La incidencia de las variables personales y socioculturales del alumno en el aprendizaje de vocabulario en español/L2. Resultados de una investigación empírica», Cauce. Revista Internacional de Filología, Comunicación y sus Didácticas, 36-37, págs. 251-273.

San-Mateo-Valdehíta, Alicia y María Antonieta Andión Herrero (2012): «Planteamiento de un proyecto de incorporación léxica en español lengua meta. Cuestiones metodológicas y estudio piloto», EPOS, XXVIII, págs. 73-90.

Sankarakumar, S.; Chandrakanth, S. y P. Malathy (2012): «The performance of male and female students of first year engineering in reading comprehension tests», Language in India, 12.5, págs. 307-324.

Schmitt, Norbert (1997): «Vocabulary learning strategies». En Schmitt, Norbert y Michael McCarthy (eds.): Vocabulary. Description, acquisition and pedagogy, 2002, 4. . ed., Cambridge, CUP, págs. 199-227.

Webb, Stuart (2005): «Receptive and productive vocabulary learning: The effects of reading and writing on word knowledge», Studies in Second Language Acquisition, 27.1, págs. 33-52.

- (2009): «The effects of receptive and productive learning of word pairs on vocabulary knowledge», Regional English Language Centre Journal, 40.3, págs. 360-376.

YAZDANPANAH, KHATEREH (2007): «The effect of background knowledge and reading comprehension test items on male and female performance», The Reading Matrix, 7.2, págs. 64-80.

ZeYnali, Simin (2012): «Exploring the gender effect on EFL learners' learning strategies», Theory and Practice in Language Studies, 2.8, págs. 1614-1620. 